OPEN ACCESS

Edited by:

Wansong Chen,

Central South University, China

Reviewed by:

Huangxian Ju,

Nanjing University, China

Saji Uthaman,

Chungnam National University,

South Korea

*Correspondence:

Yuanqiang Hao

hao0736@163.com

Specialty section:

This article was submitted to

Nanoscience,

a section of the journal

Frontiers in Chemistry

Received: 14 May 2020

Accepted: 23 July 2020

Published: 04 September 2020

Citation:

Zhang Y, Hao Y, Chen S and Xu M (2020) Photodynamic Therapy of

Cancers With Internal Light Sources:

Chemiluminescence,

Bioluminescence, and Cerenkov

Radiation. Front. Chem. 8:770

doi: 10.3389/fchem.2020.00770

\section{Photodynamic Therapy of Cancers With Internal Light Sources: Chemiluminescence, Bioluminescence, and Cerenkov Radiation}

\author{
Yintang Zhang ${ }^{1}$, Yuanqiang $\mathrm{Hao}^{1 *}$, Shu Chen ${ }^{2}$ and Maotian $\mathrm{Xu}^{1}$ \\ ${ }^{1}$ Henan Key Laboratory of Biomolecular Recognition and Sensing, College of Chemistry and Chemical Engineering, Henan \\ Joint International Research Laboratory of Chemo/Biosensing and Early Diagnosis of Major Diseases, Shangqiu Normal \\ University, Shangqiu, China, ${ }^{2}$ Key Laboratory of Theoretical Organic Chemistry and Function Molecule of Ministry of \\ Education, School of Chemistry and Chemical Engineering, Hunan University of Science and Technology, Xiangtan, China
}

Photodynamic therapy (PDT) is a promising and minimally invasive modality for the treatment of cancers. The use of a self-illuminating system as a light source provides an intriguing solution to the light penetration issues of conventional PDT, which have gained considerable research interest in the past few years. This mini review aimed to present an overview of self-illuminating PDT systems by using internal light sources (chemiluminescence, bioluminescence, and Cerenkov radiation) and to give a brief discussion on the current challenges and future perspectives.

Keywords: photodynamic therapy, cancers, internal light sources, chemiluminescence, bioluminescence, Cerenkov radiation

\section{INTRODUCTION}

Photodynamic therapy (PDT) is a promising non-invasive medical technique and has been clinically approved for treating various diseases, including bacterial and fungal infections, skin diseases, as well as several types of cancer (van Straten et al., 2017). In the past decades, advances in nanotechnology and materials science as well as the improvements of photosensitizers (PS) have promoted the rapid development of PDT (Chen et al., 2017; Glass et al., 2018; Ouyang et al., 2018a,b; Fan et al., 2019; Yue et al., 2019; Zeng et al., 2019). However, the conventional external light irradiations in PDT often suffer from rapid attenuation through the tissue, which limited the clinical use of PDT to some superficial or endoscope-accessible lesions. To overcome this barrier, different excitation sources, such as near-infrared (NIR) light and X-ray radiation, have been applied in combination with a number of judiciously designed photosensitizers, such as two-photon (Shen et al., 2016) or NIR light (Lan et al., 2019) excitation photosensitizers and upconversion photosensitizers (Liu et al., 2019). Nevertheless, it is still challenging to develop NIR photosensitizers with high absorption efficiency, and NIR light also has penetration limitation (i.e., 980-nm light is restricted to $1.5 \mathrm{~cm}$ ). X-ray radiation, photon energy in the range of $0.1-100 \mathrm{keV}$, can overcome the limitation of the light penetration depth in the human body. But X-rays can ionize atoms and disrupt chemical bonds of normal biomolecules. Implanting fiber-optic light sources could be a viable approach to treat deep tissue, but it requires invasive procedures and cannot deal with tumor metastasis. 
Internal light sources have emerged as an attractive alternative to outer light sources in a conventional PDT system for addressing the issue of light penetration (Magalhães et al., 2016; Ferreira et al., 2019). Some self-illuminating systems, including chemiluminescence (CL), bioluminescence (BL), and Cerenkov radiation (CR), are promising candidates as internal light sources for PDT as these self-illuminators are small in size (ranging from atomic/molecular to nanometer scale) and thus can be delivered to any pathological tissues. The use of self-illuminating systems as light sources in PDT has attracted increasing research interest in the past few years (Fan et al., 2016; Magalhães et al., 2016; Jiang et al., 2020). In this mini review, we summarize recent progress in the development of self-illuminating PDT systems, including chemiluminescence-, bioluminescence-, and Cerenkov radiation-mediated photodynamic therapy (Figure 1). The design strategies and chemical structures of these reported PDT systems will be emphasized. Current limitations and future directions will also be discussed.

\section{CHEMILUMINESCENCE-MEDIATED PHOTODYNAMIC THERAPY}

$\mathrm{CL}$ is a kind of self-luminescence phenomenon that results from some special types of chemical reactions. Up to now, chemiluminescence reactions have found a wide variety of applications (Iranifam, 2014; Shah et al., 2020) such as chemosensing, bioimaging, and therapy. The most commonly used chemiluminescence systems are luminol- $\mathrm{H}_{2} \mathrm{O}_{2}$ and oxalate ester- $\mathrm{H}_{2} \mathrm{O}_{2}$.

The first report of using luminol chemiluminescence as an excitation light source for PDT application was demonstrated by Laptev et al. (2006). A transferrin-hematoporphyrin (Tf-Hp) conjugate was used as the cancer cell targetable photosensitizer. The absorption spectra of Tf-Hp (one absorption peak at $412 \mathrm{~nm}$ ) overlapped well with the CL emission band of luminol (350$550 \mathrm{~nm})$. The addition of luminol can induce a noticeable intracellular CL over a 50-min period. The combination of luminol $(10 \mu \mathrm{M})$ and the Tf-Hp conjugate $(3 \mu \mathrm{M})$ resulted in 95\% cytotoxicity against the erythroleukemic cell lines.

Yuan et al. (2012) developed a new chemiluminescencemediated PDT by using a cationic oligo( $p$-phenylene vinylene) (OPV) as the photosensitizer. The cationic OPV could not only interact with the dianionic intermediates of luminol CL reaction to facilitate the occurrence of chemiluminescence resonance energy transfer (CRET) to excite OPV but could also bind to the negatively charged pathogen (cancer cells and fungi). The luminol chemiluminescence system with OPV displayed prominent cytotoxicity toward HeLa cells with a viability of $<10 \%$. Studies in tumor-bearing nude mice indicated that this self-illuminated PDT system could significantly inhibit the tumor growth in vivo without exerting apparent toxicity to the normal tissues. Based on the cationic OPV photosensitizer, the same research group proposed a novel electrochemiluminescencemediated PDT system (Liu et al., 2018). Recently, Jiang's group reported a conjugated polymer nanoparticle-based multifunctional nanoplatform for oxygen-supplying and selfilluminating phototherapy (Jiang et al., 2019). The nanoplatform $\mathrm{Hb}-\mathrm{NPs}$ was obtained by covalently coupling hemoglobin $(\mathrm{Hb})$ to the polymer nanoparticle consisting of poly(styrene-co-maleic anhydride) (PSMA) and poly[2-methoxy-5-(2-ethylhexyloxy)1,4-phenylenevinylene] (MEH-PPV). The polymer-conjugated $\mathrm{Hb}$ can simultaneously function as the oxygen carrier, the catalyst for the $\mathrm{CL}$ reaction of luminol with $\mathrm{H}_{2} \mathrm{O}_{2}$, as well as the PDT photosensitizer for generating reactive oxygen species (ROS). Thus, the presented system does not require an external light source and can overcome the problem of insufficient oxygen under hypoxia. In addition, the system can also be used to control the release of an anticancer prodrug, thus providing simultaneous phototherapy and chemotherapy.

The combination of different forms of photosensitizers with luminol CL has afforded a variety of other internal self-illuminating PDT systems, including tetra(hydroxyphenyl)chlorin (THPC)-encapsulated FH-Pdots [folic acid and horseradish peroxidase (HRP)-bifunctionalized polymer dots] (Zhang et al., 2014), 5-aminolevulinic acid (Chen et al., 2012), porphyrinic metal-organic frameworks (COTCPP MOFs) (Fang et al., 2019), chlorin e6-conjugated yellow-emissive carbon dots (y-CDs-Ce6) (Yang et al., 2020), and poly(lactic-co-glycolic acid) (PLGA)/luminol/HRP/DSPEmPEG2000 co-precipitated nanoparticles (Lu et al., 2020). Covalent attachment of luminol to the photosensitizer into a single molecule is an additional strategy for constructing a CL-PDT system through the direct energy transfer from luminol to the photosensitizer. Yesilgul et al. (2017) designed a modular unimolecular erythrosine-luminol which can produce singlet oxygen in the presence of $\mathrm{Cu}^{2+}$ and hydrogen peroxide. $\mathrm{Xu}$ et al. (2019) synthesized a Ce6-luminol-PEG (CLP) conjugate which can self-assemble into core-shell nanoparticles. The nanoparticle can be exploited for inflammation imaging as well as for specifically killing cancer cells. In addition to the commonly used chemiluminophore of luminol, peroxyoxalate chemiluminescent (PO-CL) reaction also has the potential to supply internal light source for PDT. Romanyuk et al. (2017) developed a self-luminescing PDT strategy by using polymeric oxalate $(\mathrm{POX})$ as the substrate of the $\mathrm{PO}-\mathrm{CL}$ reaction and tetramethyl hematoporhyrin (TMHP) as both a photosensitizer and an activator for the PO-CL reaction. POX and TMHP were dispersed in dimethyl phthalate (DMP) droplets and stabilized with a surfactant of Pluronic L64. The L64/DMP/POX/TMHP dispersions can effectively produce $\mathrm{O}_{2}^{1}$ and exert significant cytotoxicity under oxidative stress condition. Mao et al. (2017) designed a novel nanoplatform [C-TBD nanoparticles (NPs)] for image-guided PDT by co-encapsulating bis[2,4,5trichloro-6-(pentyloxycarbonyl)phenyl]oxalate (CPPO) and the photosensitizer TBD into an amphiphilic copolymer Pluronic F-127. The astutely designed photosensitizer TBD displayed a bright aggregation-induced NIR emission and an efficient singlet oxygen generation. C-TBD NPs could precisely monitor the tumor site via chemiluminescence imaging and efficiently inhibit tumor growth through PO-CL exciting TBD to produce $\mathrm{O}_{2}^{1}$ in the presence of $\mathrm{H}_{2} \mathrm{O}_{2}$, thus realizing simultaneous tumor diagnosis and treatment. Coelenterazine and its analogs can 


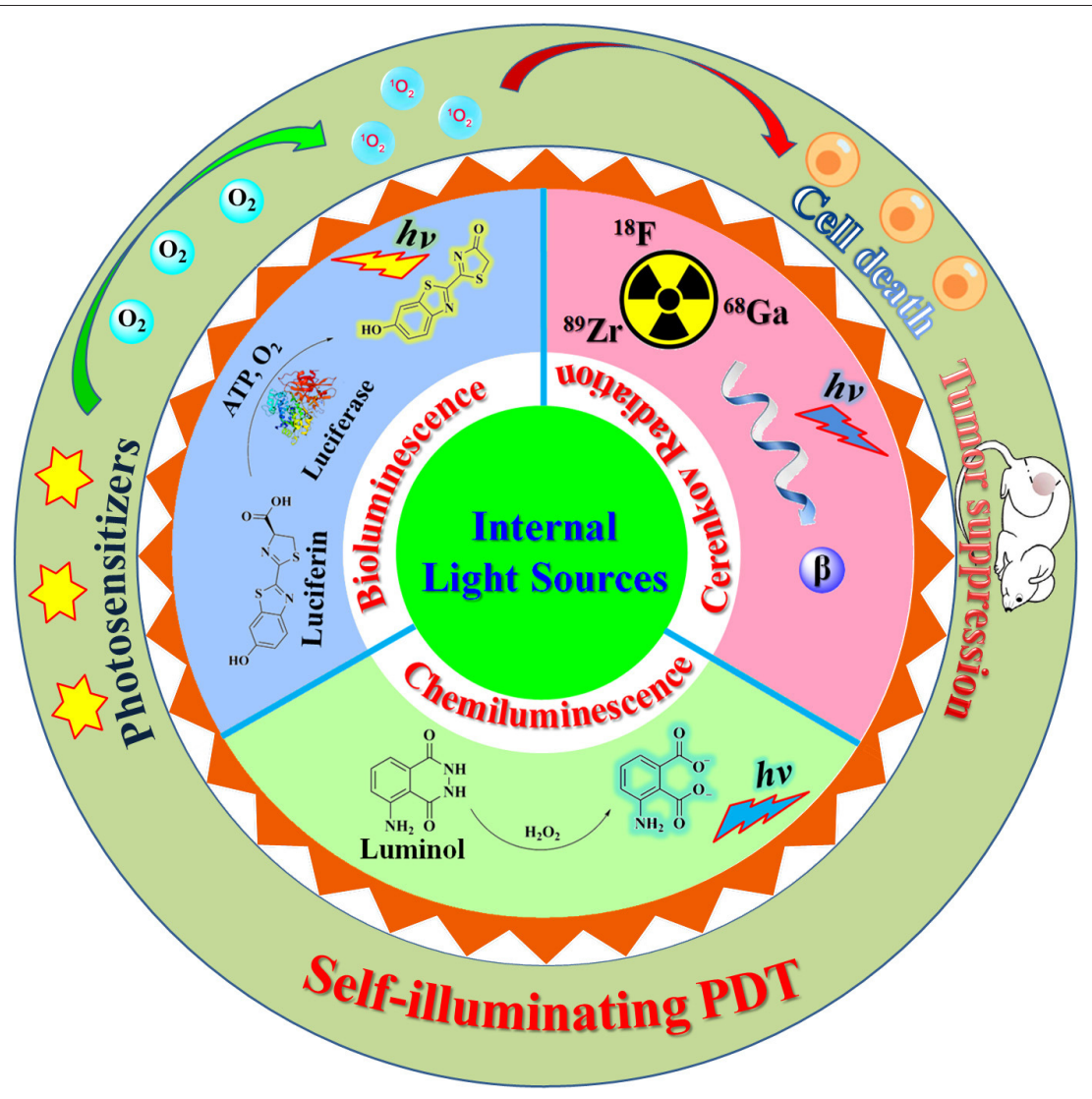

FIGURE 1 | lllustration of a self-illuminating photodynamic therapy (PDT) system based on internal light sources, including chemiluminescence, bioluminescence, and Cerenkov radiation.

also act as a self-activating agent for PDT (Pinto da Silva et al., 2019; Sun et al., 2019). In the presence of a superoxide anion (overexpressed in tumor cells), coelenterazine can be oxidized into a dioxetanone intermediate, which then decomposing into $\mathrm{CO}_{2}$ and the excited coelenteramide. The product will further undergo intersystem crossing to the triplet state, thus inducing the generation of $\mathrm{O}_{2}^{1}$. This process can be enhanced by the introduction of a bromine heteroatom due to the heavy atom effect (Pinto da Silva et al., 2019).

\section{BIOLUMINESCENCE-MEDIATED PHOTODYNAMIC THERAPY}

Bioluminescence can be considered as a special type of CL which involves the enzymatic oxidation of a small molecule substrate, such as the firefly luciferase-luciferin, and Renilla luciferasecoelenterazine systems (Magalhães et al., 2016; Hananya and Shabat, 2017). Theodossiou et al. firstly evaluated the potential of intracellular bioluminescence as a light source for PDT (Theodossiou et al., 2003). The classical firefly luciferin-luciferase system was used to excite the photosensitizer, Rose Bengal (RB), which has a high singlet oxygen quantum yield and a compatible absorption profile with the emission of oxyluciferin. Treating the luciferase-transfected NIH 3T3 cells with RB and D-luciferin led to a high rate of apoptosis (89\%). This preliminary in vitro study demonstrated the possibility of a bioluminescence-mediated PDT to be a plausible treatment modality. However, the following work by Schipper et al. (2006) revealed that luciferase-expressing cells incubated with photosensitizer and D-luciferin did not show a significant difference in survival compared with the control groups. The authors demonstrated that this intracellular bioluminescence system cannot emit enough photons $(<1.03 \times$ $10^{-4} \mathrm{~mJ} \mathrm{~cm}{ }^{-2}$ for $24 \mathrm{~h}$ treatment) to mediate photosensitizers to generate efficacious photodynamic toxicity.

In the above cases, the bioluminescent enzyme appeared in cells through gene expression, a technology that has not been applied in actual clinical treatment, so this bioluminescent system has difficulty in becoming an alternative excitation for PDT in the near future. Hsu et al. (2013) developed a new coelenterazine bioluminescence-mediated PDT system based on the bioluminescent QD-Rluc8 conjugate which was obtained by immobilizing Renilla luciferase onto quantum dot 655. In the presence of coelenterazine, QD-Rluc8 can display the distinct fluorescence emission of quantum dots (QDs) via bioluminescent resonance energy transfer (BRET) from the bioluminescent substrate to the QDs. Both in vitro and in vivo assays demonstrated that the bioluminescent QDRluc8 can stimulate the micelle-loaded photosensitizer metatetra(hydroxyphenyl)chlorin (m-THPC, Foscan) to produce ROS 
and thus result in cell death and inhibition of tumor growth. As a potential alternative excitation source for PDT, QDRluc8 displayed several advantages, including a tunable emission wavelength and a relatively high irradiation dose $(0.6-0.8 \mathrm{~J}$ $\mathrm{cm}^{-2}$ ). Subsequently, Kim et al. (2015) evaluated whether BL can induce efficient PDT for tumors, especially in deep tissue. A similar BRET Luc-QD luminophore was employed as the BL source, and Ce6 was used as the photosensitizer. Confocal imaging showed that Luc-QD conjugates were mainly distributed on the cell surface instead of in the cytoplasm. Calculating from the emission spectra, the BRET efficiency of the Luc-QD system reached $60-65 \%$. Luc-QD can excite the nearby photosensitizer, leading to an appreciable amount of activated Ce6 $\left(3 \times 10^{8} / \mathrm{min}\right)$, which is higher than that $\left(4 \times 10^{7} / \mathrm{min}\right)$ resulting from laser illumination $\left(2.2 \mathrm{~mW} / \mathrm{cm}^{2}\right)$. Cell imaging and cytotoxicity tests revealed that this BL-PDT system can generate significant intracellular ROS, thus resulting in membrane damage and cell death. Intravenous injection of Ce6, Luc-QD, and CTZ to tumorimplant mice can almost completely inhibit tumor growth. Furthermore, this BL-PDT can also suppress distant organ metastasis. Recently, Yang et al. (2018) reported a new BL-PDT system based on the polymer nanoparticle which was assembled from poly(lactic acid) and poly(lactic-co-glycolic acid) and was loaded with Rose Bengal and luciferase. In vitro photodynamic studies showed that this BL-PDT treatment can lead to significant toxicity toward cancer cells. In vivo, BL-PDT displayed that the growth of subcutaneous tumors can be remarkably inhibited, while normal organs, including the heart, liver, spleen, lung, and kidney, remained undamaged.

\section{CERENKOV RADIATION-INDUCED PHOTODYNAMIC THERAPY}

Cerenkov radiation (CR) is a luminescence phenomenon occurring from the interaction between high-speed charged particles (faster than the phase velocity of light) with the surrounding medium, which was theoretically predicted by Heaviside in 1888 and experimentally verified by Cerenkov in 1933 (Shaffer et al., 2017). In biomedical applications, the commonly used sources for producing Cerenkov radiation are radionuclides which can emit $\beta$ particles (positrons or electrons) (Gill et al., 2015), such as ${ }^{18} \mathrm{~F},{ }^{64} \mathrm{Cu},{ }^{68} \mathrm{Ga},{ }^{89} \mathrm{Zr},{ }^{90} \mathrm{Y},{ }^{124} \mathrm{I}$, and ${ }^{198} \mathrm{Au}$. The number of Cerenkov photons within a certain wavelength range is positively correlated to the kinetic energy (or velocity) of the $\beta$ particle. Radionuclides emitting $\beta$ particles with relatively higher energy, such as ${ }^{68} \mathrm{Ga}$ and ${ }^{90} \mathrm{Y}$, would produce CR with higher intensity (Klein et al., 2019). In recent years, the Cerenkov phenomenon for biomedical applications (i.e., imaging and therapy) has gradually garnered significant attention, especially combining the rapid advancements in nanosciences and nanotechnologies (Shaffer et al., 2017; Cline et al., 2019; Ferreira et al., 2019).

In 2015, Kotagiri and coworkers reported a proof-ofconcept study using $\mathrm{CR}$ from a common diagnostic clinical radiotracer, $2^{\prime}$-deoxy- $2^{\prime}-\left[{ }^{18} \mathrm{~F}\right]$ fluoro-D-glucose $\left(\left[{ }^{18} \mathrm{~F}\right]-\mathrm{FDG}\right)$, as the light source for PDT, denoted as CR-induced therapy (CRIT)
(Kotagiri et al., 2015). By incorporating both apotransferrin (Tf) and titanocene (Tc) into $\mathrm{TiO}_{2}$ nanoparticles, the authors designed and prepared an effective nanophotosensitizer $\left(\mathrm{TiO}_{2}\right.$ Tf-Tc) which can function with low-intense radiation. $\mathrm{TiO}_{2}$ nanoparticles have the capability to effectively utilize $\mathrm{CR}$, which locates predominantly within the ultraviolet to the blue spectral region (characteristic $1 / \lambda^{2}$ spectrum), to generate hydroxyl and superoxide radicals. Tf can not only act as a stabilizer and dispersant for $\mathrm{TiO}_{2}$ but also as a tumor-targeting agent. Tc can serve as a complementary photosensitizer for generating cyclopentadienyl and titanium-centered radicals. In vitro cellular experiments have shown that $\mathrm{TiO}_{2}$-Tf can be endocytosed by tumor cells and, together with $\left[{ }^{18} \mathrm{~F}\right]-\mathrm{FDG}$, can lead to an appreciable decrease in cell viability. In vivo studies have demonstrated that the administrated $\mathrm{TiO}_{2}$-Tf-Tc prefer to accumulate in the tumor tissue and that the co-administration of $\mathrm{TiO}_{2}$ - Tf- $\mathrm{Tc}$ and radionuclides efficiently inhibited the tumor growth and increased the median survival. These results demonstrated the possibility of utilizing $\mathrm{CR}$ as the internal exciting light for PDT photosensitizers, and this pioneering work opened a new avenue for the application of the Cerenkov effect in PDT, especially in a depth- and oxygen-independent manner. Following this study, Duan et al. (2018) reported another CRIT system by using ${ }^{68} \mathrm{Ga}$-labeled bovine serum albumin $\left({ }^{68} \mathrm{Ga}-\mathrm{BSA}\right)$ as a more efficient Cherenkov radiation emitter and dextran-modified $\mathrm{TiO}_{2}$ nanoparticles $\left(\mathrm{D}-\mathrm{TiO}_{2} \mathrm{NPs}\right)$ as the nanophotosensitizer. For comparison, $\left[{ }^{18} \mathrm{~F}\right]$-FDG was also used throughout this study. Positron emission tomography (PET) images of tumor-bearing mice intratumorally injected with radionuclides revealed that both ${ }^{68} \mathrm{Ga}-\mathrm{BSA}$ and $\left[{ }^{18} \mathrm{~F}\right]-\mathrm{FDG}$ can predominantly stay in the tumor in similar amounts, whereas much more CR photon accumulation appeared in mouse loaded with ${ }^{68} \mathrm{Ga}$-BSA. As a result, ${ }^{68} \mathrm{Ga}$-BSA with $\mathrm{D}-\mathrm{TiO}_{2}$ exhibited a significantly enhanced PDT efficacy. Injection of ${ }^{68} \mathrm{Ga}$-BSA and $\mathrm{D}-\mathrm{TiO}_{2} \mathrm{NPs}$ into the tumor mass caused complete suppression of the tumor growth and a significant increase in median survival, while no obvious efficacy was observed for the other group, even with the combination of $\left[{ }^{18} \mathrm{~F}\right]-\mathrm{FDG}$ and $\mathrm{D}-\mathrm{TiO}_{2} \mathrm{NPs}$.

In the above two studies, the Cerenkov source (radiotracer) and the PDT photosensitizer were used individually, as two detached elements. After administration, the radiotracer and photosensitizer would randomly distribute in the target location and within a relatively large distance. Thus, a large portion of the Cerenkov photons from the radiotracer may have probably been absorbed or scattered by the media instead of exciting the photosensitizer. To circumvent this problem, Kamkaew et al. (2016) designed an integrated CRIT system, [ $\left.{ }^{89} \mathrm{Zr}\right]$ HMSN-Ce6, by loading the Cerenkov source $\left({ }^{89} \mathrm{Zr}\right)$ and photosensitizer (Ce6) into hollow mesoporous silica nanoparticles (HMSNs). In vitro imaging displayed that $\left[{ }^{89} \mathrm{Zr}\right] \mathrm{HMSN}-\mathrm{Ce} 6$ can emit intense fluorescence $(690-710 \mathrm{~nm})$ in solution without external excitation light, which clearly confirmed the occurrence of energy transfer between ${ }^{89} \mathrm{Zr}$ and Ce6. In vivo CR-induced PDT studies further confirmed the efficacy of $\left[{ }^{89} \mathrm{Zr}\right]$ HMSN-Ce6 to completely inhibit tumor growth. Subsequently, the same research group proposed a magnetic targetable CRIT system (Ni et al., 2018), ${ }^{89} \mathrm{Zr}-\mathrm{MNP} / \mathrm{TCPP}$, which was prepared by 
labeling PEGylated magnetic nanoparticles (MNP) with ${ }^{89} \mathrm{Zr}$ via Lewis acid-base interaction and then conjugating with the photosensitizer, meso-tetrakis(4-carboxyphenyl)porphyrin (TCPP), via amide coupling. Various imaging analyses (including PET, fluorescence, CL, and CRET) confirmed that the magnetic nanoparticles can be accumulated in tumor regions under an external magnetic field. The ${ }^{89} \mathrm{Zr}$-MNP/TCPP ternary composite has shown efficient ${ }^{1} \mathrm{O}_{2}$ generation efficiency as well as a high magnetic-guided therapeutic efficacy. Recently, Yu's group proposed a novel "missile detonation" strategy for efficient CR-induced theranostics by the successive administration of a high dose of porphyrin-PEG nanocomplex (PPN) and a low dose of ${ }^{89} \mathrm{Zr}$-labeled PPN ( ${ }^{89} \mathrm{Zr}$-Df-PPN) (Yu et al., 2019). The PPN and ${ }^{89} \mathrm{Zr}$-Df-PPN acted as the CR energy receiver/missile and ${ }^{89} \mathrm{Zr}$-Df-PPN the CR energy donor/detonator, respectively. These therapeutic agents can be monitored via multimodal imaging, including fluorescence imaging, CRET imaging, and PET. This CRIT system could significantly inhibit tumor growth in vivo and cause substantial fragmentation of vascular in the tumor.

\section{CONCLUSIONS AND PERSPECTIVES}

In this review, we have summarized recent advances in the development of self-illuminating PDT systems. Internal light sources, including chemiluminescence, bioluminescence, and Cerenkov radiation, have been proven capable of exciting certain photosensitizers to produce ROS and generate efficacious photodynamic toxicity. The use of these internal light sources has great potential for depth-independent PDT, which would expand the scope of PDT in cancer treatment.

Despite the impressive progress outlined above, the current self-illuminating PDT systems still face several challenges, including a relatively low photon flux, the requirement of multicomponent reactions for $\mathrm{CL}$ and $\mathrm{BL}$, limited wavelength range of the excitation light, etc. To overcome these shortcomings, several future directions for the design of self-illuminating PDT systems are proposed as follows:

(1) Nanocarrier technologies can be employed for increasing the amount of administrated self-illuminators. If the reactants for producing internal light, such as luminol, luciferase-luciferin pairs, and CR radiotracer, were loaded and delivered to the tumor tissues by proper nanocarriers, they would provide a higher local photon flux and, thus, more efficient PDT. The

\section{REFERENCES}

Chen, T.-C., Huang, L., Liu, C.-C., Chao, P.-J., and Lin, F.-H. (2012). Luminol as the light source for in situ photodynamic therapy. Process Biochem. 47, 1903-1908. doi: 10.1016/j.procbio.2012.06.025

Chen, W., Ouyang, J., Liu, H., Chen, M., Zeng, K., Sheng, J., et al. (2017). Black phosphorus nanosheet-based drug delivery system for synergistic photodynamic/photothermal/chemotherapy of cancer. Adv. Mater. 29:1603864. doi: 10.1002/adma.201603864

Cline, B., Delahunty, I., and Xie, J. (2019). Nanoparticles to mediate X-ray-induced photodynamic therapy and Cherenkov radiation photodynamic therapy. WIREs Nanomed. Nanobiotechnol. 11:e1541. doi: 10.1002/wnan.1541 use of multifunctional nanoplatforms for a targeted delivery and controlled release of these illuminators is also recommended.

(2) Nanophotosensitizers are more suitable for selfilluminating PDT systems as they (metal oxide nanocrystals, QDs, polymer dots, etc.) have higher cross-sections for absorbing light compared with single small-molecule photosensitizers. Moreover, nanophotosensitizers, such as QDs, can be tuned to absorb light with wavelengths ranging from near-UV through visible to near-IR.

(3) The currently used internal light sources can be further expanded. Compared with the conventional CL systems of luminol- $\mathrm{H}_{2} \mathrm{O}_{2}$ and oxalate ester $-\mathrm{H}_{2} \mathrm{O}_{2}$, the newly developed triggerable dioxetanes are more attractive chemiluminescent probes (Hananya and Shabat, 2017) as they have tunable structures and emission features, high $\mathrm{CL}$ quantum yields, without the requirement of an enzyme/catalase and $\mathrm{H}_{2} \mathrm{O}_{2}$. Thus, triggerable dioxetanes hold great potential as internal light sources for PDT. The use of a structure-modified luciferin as the substrate is a desirable approach for tuning the emission wavelength of $\mathrm{BL}$, which would expand the application of $\mathrm{BL}$ in PDT.

(4) The combined theranostic strategy is also an exciting trend for improving the efficacy of self-illuminating PDT as the integration of two or more treatments can generate synergistic effects for antitumor therapy. Thus, a promising future direction is to combine self-illuminating PDT with various other treatments, such as immunotherapy, chemotherapy, and photothermal therapy.

\section{AUTHOR CONTRIBUTIONS}

$\mathrm{YH}$ and $\mathrm{YZ}$ organized and wrote the manuscript. SC and MX discussed the results. All authors approved this manuscript.

\section{FUNDING}

This work was financially supported by the National Natural Science Foundation of China (Grant No. 21804085).

\section{ACKNOWLEDGMENTS}

This is a short text to acknowledge the contributions of specific colleagues, institutions, or agencies that aided the efforts of the authors. 
elimination of deep-seated tumor cells. J. Solid State Chem. 276, 205-209. doi: 10.1016/j.jssc.2019.05.015

Ferreira, C. A., Ni, D., Rosenkrans, Z. T., and Cai, W. (2019). Radionuclide-activated nanomaterials and their biomedical applications. Angew. Chem. Int. Ed. 58, 13232-13252. doi: 10.1002/anie.2019 00594

Gill, R. K., Mitchell, G. S., and Cherry, S. R. (2015). Computed cerenkov luminescence yields for radionuclides used in biology and medicine. Phys. Med. Biol. 60, 4263-4280. doi: 10.1088/0031-9155/60/11/4263

Glass, S., Trinklein, B., Abel, B., and Schulze, A. (2018). TiO2 as photosensitizer and photoinitiator for synthesis of photoactive TiO2-PEGDA hydrogel without organic photoinitiator. Front. Chem. 6:340. doi: 10.3389/fchem.2018.00340

Hananya, N., and Shabat, D. (2017). A glowing trajectory between bio- and chemiluminescence: from luciferin-based probes to triggerable dioxetanes. Angew. Chem. Int. Ed. 56, 16454-16463. doi: 10.1002/anie.201706969

Hsu, C.-Y., Chen, C.-W., Yu, H.-P., Lin, Y.-F., and Lai, P.-S. (2013). Bioluminescence resonance energy transfer using luciferase-immobilized quantum dots for self-illuminated photodynamic therapy. Biomaterials 34, 1204-1212. doi: 10.1016/j.biomaterials.2012.08.044

Iranifam, M. (2014). Analytical applications of chemiluminescence methods for cancer detection and therapy. TrAC Trends Anal. Chem. 59, 156-183. doi: 10.1016/j.trac.2014.03.010

Jiang, L., Bai, H., Liu, L., Lv, F., Ren, X., and Wang, S. (2019). Luminescent, oxygen-supplying, hemoglobin-linked conjugated polymer nanoparticles for photodynamic therapy. Angew. Chem. Int. Ed. 58, 10660-10665. doi: $10.1002 /$ anie. 201905884

Jiang, L., Liu, L., Lv, F., Wang, S., and Ren, X. (2020). Integration of self-luminescence and oxygen self-supply: a potential photodynamic therapy strategy for deep tumor treatment. Chempluschem 85, 510-518. doi: $10.1002 /$ cplu.202000083

Kamkaew, A., Cheng, L., Goel, S., Valdovinos, H. F., Barnhart, T. E., Liu, Z., et al. (2016). Cerenkov radiation induced photodynamic therapy using chlorin e6loaded hollow mesoporous silica nanoparticles. ACS Appl. Mater. Interfaces 8, 26630-26637. doi: 10.1021/acsami.6b10255

Kim, Y. R., Kim, S., Choi, J. W., Choi, S. Y., Lee, S.-H., Kim, H., et al. (2015). Bioluminescence-activated deep-tissue photodynamic therapy of cancer. Theranostics 5, 805-817. doi: 10.7150/thno.11520

Klein, J. S., Sun, C., and Pratx, G. (2019). Radioluminescence in biomedicine: physics, applications, and models. Phys. Med. Biol. 64:04TR01. doi: 10.1088/1361-6560/aaf4de

Kotagiri, N., Sudlow, G. P., Akers, W. J., and Achilefu, S. (2015). Breaking the depth dependency of phototherapy with cerenkov radiation and lowradiance-responsive nanophotosensitizers. Nat. Nanotechnol. 10, 370-379. doi: 10.1038/nnano.2015.17

Lan, M., Zhao, S., Liu, W., Lee, C.-S., Zhang, W., and Wang, P. (2019). Photosensitizers for photodynamic therapy. Adv. Healthcare Mater. 8:1900132. doi: 10.1002/adhm.201900132

Laptev, R., Nisnevitch, M., Siboni, G., Malik, Z., and Firer, M. A. (2006). Intracellular chemiluminescence activates targeted photodynamic destruction of leukaemic cells. Br. J. Cancer 95, 189-196. doi: 10.1038/sj.bjc.6603241

Liu, S., Yuan, H., Bai, H., Zhang, P., Lv, F., Liu, L., et al. (2018). Electrochemiluminescence for electric-driven antibacterial therapeutics. J. Am. Chem. Soc. 140, 2284-2291. doi: 10.1021/jacs.7b12140

Liu, Y., Meng, X., and Bu, W. (2019). Upconversion-based photodynamic cancer therapy. Coord. Chem. Rev. 379, 82-98. doi: 10.1016/j.ccr.2017.09.006

Lu, X., Song, X., Wang, Q., Hu, W., Shi, W., Tang, Y., et al. (2020). Chemiluminescent organic nanophotosensitizer for a penetration depth independent photodynamic therapy. RSC Adv. 10, 11861-11864. doi: 10.1039/D0RA01477]

Magalhães, C. M., Esteves da Silva, J. C. G., and Pinto da Silva, L. (2016). Chemiluminescence and bioluminescence as an excitation source in the photodynamic therapy of cancer: a critical review. Chemphyschem 17, 2286-2294. doi: 10.1002/cphc.201600270

Mao, D., Wu, W., Ji, S., Chen, C., Hu, F., Kong, D., et al. (2017). Chemiluminescence-guided cancer therapy using a chemiexcited photosensitizer. Chem 3, 991-1007. doi: 10.1016/j.chempr.2017. 10.002

Ni, D. L., Ferreira, C. A., Barnhart, T. E., Quach, V., Yu, B., Jiang, D. W., et al. (2018). Magnetic targeting of nanotheranostics enhances cerenkov radiation-induced photodynamic therapy. J. Am. Chem. Soc. 140, 14971-14979. doi: 10.1021/jacs.8b09374

Ouyang, J., Deng, Y., Chen, W., Xu, Q., Wang, L., Liu, Z., et al. (2018a). Marriage of artificial catalase and black phosphorus nanosheets for reinforced photodynamic antitumor therapy. J. Mater. Chem. B 6, 2057-2064. doi: $10.1039 / \mathrm{C} 8 \mathrm{~TB} 00371 \mathrm{H}$

Ouyang, J., Wang, L., Chen, W., Zeng, K., Han, Y., Xu, Y., et al. (2018b). Biomimetic nanothylakoids for efficient imaging-guided photodynamic therapy for cancer. Chem. Commun. 54, 3468-3471. doi: 10.1039/C8CC00674A Pinto da Silva, L., Núnez-Montenegro, A., Magalhães, C. M., Ferreira, P. J. O., Duarte, D., González-Berdullas, P., et al. (2019). Single-molecule chemiluminescent photosensitizer for a self-activating and tumorselective photodynamic therapy of cancer. Eur. J. Med. Chem. 183:111683. doi: 10.1016/j.ejmech.2019.111683

Romanyuk, A. V., Grozdova, I. D., Ezhov, A. A., and Melik-Nubarov, N. S. (2017). Peroxyoxalate chemiluminescent reaction as a tool for elimination of tumour cells under oxidative stress. Sci. Rep. 7:3410. doi: 10.1038/s41598-017-03527-w

Schipper, M. L., Patel, M. R., and Gambhir, S. S. (2006). Evaluation of firefly luciferase bioluminescence mediated photodynamic toxicity in cancer cells. Mol. Imaging Biol. 8, 218-225. doi: 10.1007/s11307-006-0048-1

Shaffer, T. M., Pratt, E. C., and Grimm, J. (2017). Utilizing the power of cerenkov light with nanotechnology. Nat. Nanotechnol. 12, 106-117. doi: 10.1038/nnano.2016.301

Shah, S. N. A., Khan, M., and Rehman, Z. U. (2020). A prolegomena of periodate and peroxide chemiluminescence. TrAC Trends Anal. Chem. 122:115722. doi: $10.1016 /$ j.trac.2019.115722

Shen, Y., Shuhendler, A. J., Ye, D., Xu, J.-J., and Chen, H.-Y. (2016). Twophoton excitation nanoparticles for photodynamic therapy. Chem. Soc. Rev. 45 , 6725-6741. doi: 10.1039/C6CS00442C

Sun, S.-K., Wu, J.-C., Wang, H., Zhou, L., Zhang, C., Cheng, R., et al. (2019). Turning solid into gel for high-efficient persistent luminescence-sensitized photodynamic therapy. Biomaterials 218:119328. doi: 10.1016/j.biomaterials.2019.119328

Theodossiou, T., Hothersall, J. S., Woods, E. A., Okkenhaug, K., Jacobson, J., and MacRobert, A. J. (2003). Firefly luciferin-activated rose bengal. Cancer Res. 63:1818.

van Straten, D., Mashayekhi, V., de Bruijn, H. S., Oliveira, S., and Robinson, D. J. (2017). Oncologic photodynamic therapy: basic principles, current clinical status and future directions. Cancers 9:19. doi: 10.3390/cancers9020019

Xu, X., An, H., Zhang, D., Tao, H., Dou, Y., Li, X., et al. (2019). A self-illuminating nanoparticle for inflammation imaging and cancer therapy. Sci. Adv. 5:eaat2953. doi: 10.1126/sciadv.aa t2953

Yang, K., Wang, C., Wei, X., Ding, S., Liu, C., Tian, F., et al. (2020). Self-illuminating photodynamic therapy with enhanced therapeutic effect by optimization of the chemiluminescence resonance energy transfer step to the photosensitizer. Bioconjugate Chem. 31, 595-604. doi: 10.1021/acs.bioconjchem.9b00740

Yang, Y., Hou, W., Liu, S., Sun, K., Li, M., and Wu, C. (2018). Biodegradable polymer nanoparticles for photodynamic therapy by bioluminescence resonance energy transfer. Biomacromolecules 19, 201-208. doi: 10.1021/acs.biomac.7b01469

Yesilgul, N., Uyar, T. B., Seven, O., and Akkaya, E. U. (2017). Singlet oxygen generation with chemical excitation of an erythrosine-luminol conjugate. ACS Omega 2, 1367-1371. doi: 10.1021/acsomega.7b00228

Yu, B., Ni, D., Rosenkrans, Z. T., Barnhart, T. E., Wei, H., Ferreira, C. A., et al. (2019). A "missile-detonation" strategy to precisely supply and efficiently amplify cerenkov radiation energy for cancer theranostics. Adv. Mater. 31:1904894. doi: 10.1002/adma.201904894

Yuan, H., Chong, H., Wang, B., Zhu, C., Liu, L., Yang, Q., et al. (2012). Chemical molecule-induced light-activated system for anticancer and 
antifungal activities. J. Am. Chem. Soc. 134, 13184-13187. doi: 10.1021/ja30 $4986 \mathrm{t}$

Yue, J., Liang, L., Shen, Y., Guan, X., Zhang, J., Li, Z., et al. (2019). Investigating dynamic molecular events in melanoma cell nucleus during photodynamic therapy by SERS. Front. Chem. 6:665. doi: 10.3389/fchem.2018. 00665

Zeng, K., Xu, Q., Ouyang, J., Han, Y., Sheng, J., Wen, M., et al. (2019). Coordination nanosheets of phthalocyanine as multifunctional platform for imaging-guided synergistic therapy of cancer. ACS Appl. Mater. Interfaces 11, 6840-6849. doi: 10.1021/acsami.8b22008

Zhang, Y., Pang, L., Ma, C., Tu, Q., Zhang, R., Saeed, E., et al. (2014). Small molecule-initiated light-activated semiconducting polymer dots: an integrated nanoplatform for targeted photodynamic therapy and imaging of cancer cells. Anal. Chem. 86, 3092-3099. doi: 10.1021/ac404201s
Conflict of Interest: The authors declare that the research was conducted in the absence of any commercial or financial relationships that could be construed as a potential conflict of interest.

The handling editor declared a past co-authorship with the authors YZ, YH, SC, MX.

Copyright (c) 2020 Zhang, Hao, Chen and Xu. This is an open-access article distributed under the terms of the Creative Commons Attribution License (CC BY). The use, distribution or reproduction in other forums is permitted, provided the original author(s) and the copyright owner(s) are credited and that the original publication in this journal is cited, in accordance with accepted academic practice. No use, distribution or reproduction is permitted which does not comply with these terms. 